\title{
Inversion of the Odd-Even Effect in Cold Fission from the Time- Dependent Pairing Equations
}

\author{
M. Mirea ${ }^{1, a}$ \\ ${ }^{1}$ Horia Hulubei National Institute for Physics and Nuclear Engineering, P.O. Box MG-G, Bucharest, Romania
}

\begin{abstract}
A peculiar phenomenon was observed experimentally in cold fission: the odd partition yields are favored over the even ones for excitations energies of the fragments smaller than $4 \mathrm{MeV}$. In this contribution, a microscopic model is proposed for the explanation of this odd-even effect in cold fission. This explanation is based on a mixing configuration mechanism that is produced during the fission process. This configuration mixing mechanism is obtained dynamically by solving a the generalized system of time-dependent pairing equations, which include a pair-breaking effect. The time dependent equations of motion for the pair breaking effect were corroborated with a condition that fixes dynamically the number of particles on the two fission fragment. The single particle level scheme was calculated with the Woods-Saxon superasymmetric two center shell model, providing a continuous variation of the single particle energies and of the wave functions from one nucleus up to two separated fragments. A first rule can be extracted from this model. The even-even fission products cannot be obtained at zero excitation energies because of the existence of dynamical excitations produced in the avoided- level-crossing regions when the nuclear system deforms slowly.
\end{abstract}

\section{Introduction}

There are some strange experimental evidences in low energy fission. In the thermal neutron induced fission, the mass and charge distributions of fragments exhibit a fine structure that show a preference for even neutrons and protons fission products [1-5]. We expect that this phenomenon will be more evidenced in the case of cold fission, where the excitation energy of the fragments is so small that no neutrons can be emitted. This is not true as evidenced experimentally [6, 7]. For example, in the case of the mass fragmentation $(132 / 104)$ for the reaction ${ }^{235} \mathrm{U}\left(\mathrm{n}_{t h}, \mathrm{f}\right)$ in the cold fission regime, the even-even charge split (50/42) can be observed up to a total kinetic energy several MeV lower than the $Q$-value $(206 \mathrm{MeV})$. That means, the final fission products are released with at least several $\mathrm{MeV}$ excitation energy. In the same time, the odd-odd fragmentation (51/41) has yields that amount a total kinetic energy close to the $Q$-value $(202 \mathrm{MeV})$. It is strange, but at very low excitation energy an inversion of the odd-even effect is produced and the odd-odd fragmentation dominates. The eveneven fragmentation dominates at larger excitation energies while the odd-odd one dominates at very low excitation energies. This behavior is typical, being observed also for the fission of $\mathrm{Cf}[8,9]$. This inversion can be explained statistically by involving the level densities of odd and even nuclei

\footnotetext{
a e-mail: mirea@ifin.nipne.ro
} 


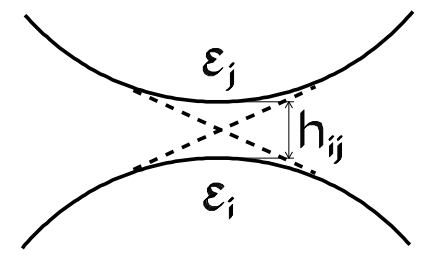

$[10,11]$. This effect is also linked to the important question of intrinsic excitation (quasiparticle excitations) during the dynamical evolution of the fissioning nucleus on ints way from ground state to scission. Another explanation can be based on the Landau-Zener promotion mechanism during fission [12] or at scission [13].

\section{The Landau-Zener effect}

The single particles or potential levels characterized by the same good quantum numbers associated to some symmetries of the system cannot in general intersect and exhibit avoided level crossing regions. An interaction $h_{i j}$ is always present in these regions between two single particle levels $\epsilon_{i}$ and $\epsilon_{j}$. Such an ideal avoided level crossing is plotted in figure 1 as function of a deformation parameter of the nuclear system, that could be the internuclear distance between the two nascent fragments. If the system deforms slowly in time and a nucleon is located on the orbital $\epsilon_{i}$, the nucleon will remain on the same adiabatic level after the passage of the avoided crossing region. If the deformation parameter varies rapidly, the nucleon will skip with a large probability from one adiabatic level to the other. It is possible to know the final probabilities of occupation of a single particle level after the passage through the avoided crossing level region by solving a set of time dependent coupled channel equations. These equations are derived from the time dependent Schrödinger equation. In general the Landau-Zener effect describes the non adiabatic transitions in avoided level crossing regions between potential curves or energy levels.

\section{Mixing of seniority zero and seniority two configurations}

It will be interesting to investigate the behavior of this effect in the case of superfluid systems, where each level is filled with some probabilities by a Cooper pair. Due to the interaction in the avoided crossing regions, it is possible that only one nucleon of the pair skips to the another level producing a dynamical quasiparticle excitation. Three situations depicted in figure 2 are possible. In the first case, the pairs remain on the same single particle level after the passage through the avoided level crossing region. In the second case, a pair destruction is illustrated. In the last case, two fermions generates a pair. A formalism is elaborated to describe these situations by using quasiparticle creation $\alpha_{i}^{+}$and annihilation operators $\alpha_{i}$. Within such operators, we construct some interactions able to break or to 


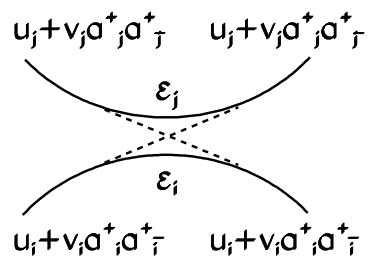

(a)

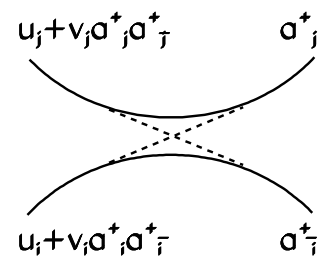

(b)

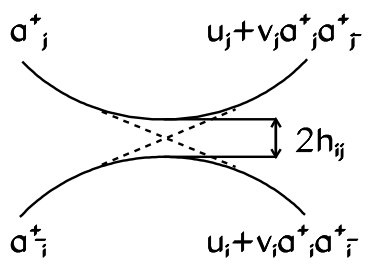

(c)

Figure 2. (a) The configuration remains unchanged after the passage through the avoided crossing region; (b) A pair is broken; (c) A pair is created.

create a pair when the system traverses an avoided level crossing region. The second situation can be obtained with an operator of the type $\alpha_{i} \alpha_{j}$ that acts on a Bogoliubov wave function, two particles being obtained on the levels $i$ and $j$. The third situation is obtained with operators $\alpha_{i}^{+} \alpha_{j}^{+}$acting on a seniority two configuration, a pair being initially distributed on the levels $i$ and $j$. This kind of perturbations were postulated in a previous work where the Landau-Zener effect was generalized in the superfluid seniority one systems [14]. Using creation and annihilation operators, the equations that governs the pair breaking mechanism are obtained from the variational principle [15, 16]. A residual perturbation in the avoided level crossing region is postulated as follows:

$$
\begin{aligned}
H^{\prime}(t)=\sum_{i, j \neq i}^{n} h_{i j}(t) & {\left[\alpha_{i(0)} \alpha_{\bar{j}(0)} \prod_{k \neq i, j} \alpha_{k(0)} \alpha_{k(i j)}^{+}\right.} \\
& \left.+\alpha_{i(0)}^{+} \alpha_{\bar{j}(0)}^{ \pm} \prod_{k \neq i, j} \alpha_{k(i j)} \alpha_{k(0)}^{+}\right],
\end{aligned}
$$

where $h_{i j}$ is the interaction energy between levels.

Another problem is that the two fission products must be characterized by integer numbers of neutrons and protons. As a consequence, the sums of the occupation probabilities of single particle levels of both fragments must give integer mass and charge numbers. By solving the equations of motion, unfortunately, the sum of single-particle densities (BCS occupation probabilities) of the single particle levels belonging to each fission fragment don't give exactly their numbers of nucleons at scission. A recipe can be implemented to fix dynamically these numbers of particles in the two final fragments by using the operators for the number of particles $\hat{N}_{i}(i=1,2)$ that act on each fission product. At scission, the two fission fragments must be characterized by a supplementary condition

$$
\left|N_{2} \hat{N}_{1}-N_{1} \hat{N}_{2}\right|=0
$$

where $N_{1}$ and $N_{2}$ are the number of particles in the final fragments labeled 1 and 2, respectively, and

$$
\hat{N}_{1}=\sum_{k 1}\left(a_{k_{1}}^{+} a_{k_{1}}+a_{k_{1}}^{ \pm} a_{\bar{k}_{1}}\right) ; \quad \hat{N}_{2}=\sum_{k 2}\left(a_{k_{2}}^{+} a_{k_{2}}+a_{k_{2}}^{ \pm} a_{k_{2}}\right)
$$

are the corresponding operators. Here, $k_{1}$ and $k_{2}$ run over the pairing active level states that are located in the potential wells of the final fragment 1 and of the final fragment 2 , respectively. The condition (1) can be introduced in the equations of motion by means of the Lagrange multipliers [17, 18]. 
All the previous ingredients could be used to obtain the microscopic equations of motion. These equations are obtained from the variational principle by minimizing the following energy functional

$$
\delta \mathcal{L}=\delta\left\langle\varphi\left|H+H^{\prime}-\lambda\right| N_{2} \hat{N}_{1}-N_{1} \hat{N}_{2}\left|-i \hbar \frac{\partial}{\partial t}\right| \varphi\right\rangle .
$$

The trial many-body function $\varphi$ is a superposition of Bogoliubov seniority zero and seniority two wave functions

$$
\begin{aligned}
|\varphi(t)\rangle= & {\left[c_{0} \prod_{k}\left(u_{k(0)}(t)+v_{k(0)}(t) a_{k}^{+} a_{k}^{ \pm}\right)\right.} \\
& \left.+\sum_{j, l \neq j} c_{j l}(t) a_{j}^{+} a_{l}^{ \pm} \prod_{k \neq j, l}\left(u_{k(j l)}(t)+v_{k(j l)}(t) a_{k}^{+} a_{k}^{ \pm}\right)\right]|0\rangle .
\end{aligned}
$$

$c_{0}$ is the amplitude of the seniority zero wave function while $c_{j l}$ are amplitudes for the seniority two wave functions for configurations in which the single particle orbitals $j$ and $l$ in the active pairing space are each blocked by only one unpaired nucleon. Here, the vacancy $u_{k}$ and occupation $v_{k}$ amplitudes are not the adiabatic solutions of the BCS equations and depend on the variation in time of the generalized parameters and the history of the nuclear system. $\lambda$ is a Lagrange multiplier. The next coupled channel equations are obtained, eventually:

$$
\begin{gathered}
i \hbar \dot{\rho}_{k(0)}=\kappa_{k(0)} \Delta_{k(0)}^{*}-\kappa_{k(0)}^{*} \Delta_{k(0)}, \\
i \hbar \dot{\rho}_{k(j l)}=\kappa_{k(j l)} \Delta_{k(j l)}^{*}-\kappa_{k(j l)}^{*} \Delta_{k(j l)}, \\
i \hbar \dot{\kappa}_{k(0)}=\left(2 \rho_{k(0)}-1\right) \Delta_{k(0)}+2 \kappa_{k(0)}\left(\epsilon_{k}-s N_{i_{k}} \lambda\right) \\
-2 G_{k k} \rho_{k(0)} \kappa_{k(0)}, \\
i \hbar \dot{\kappa}_{k(j l)}=\left(2 \rho_{k(j l)}-1\right) \Delta_{k(j l)}+2 \kappa_{k(j l)}\left(\epsilon_{k}-s N_{i_{k}} \lambda\right) \\
-2 G_{k k} \rho_{k(j l)} \kappa_{k(j l)}, \\
i \hbar \dot{P}_{0}=\sum_{l, j \neq l} h_{l j}\left(S_{0 j l}^{*}-S_{0 j l}\right) \\
i \hbar \dot{P}_{j l}=h_{l j}\left(S_{0 j l}-S_{0 j l}^{*}\right) \\
i \hbar \dot{S}_{0 j l}=S_{0 j l}\left(\bar{E}_{0}-\bar{E}_{j l}\right)+S_{0 j l}\left(\sum_{k \neq j, l} T_{k(j l)}-\sum_{k} T_{k(0)}\right) \\
+\sum_{\{m n\} \neq\{j l\}} h_{m n} S_{m n j l}+h_{j l}\left(P_{j l}-P_{0}\right) \\
\bar{S}_{m n j l}=S_{m n j l}\left(\bar{E}_{m n}-\bar{E}_{j l}\right)+S_{m n j l}\left(\sum_{k \neq m, n} T_{k(m n)}-\sum_{k \neq j, l} T_{k(j l)}\right) \\
+h_{m n} S_{0 j l}-h_{j l} S_{0 m n}^{*}
\end{gathered}
$$

where $j, k, l, m, n$ label the single particle levels in the active pairing space. The sign $s= \pm 1$ ensures that the matrix element of the expression (1) is positive. $N_{i_{k}}=N_{2}$ or $N_{i_{k}}=-N_{1}$ if the state $k$ will be located in the fragment 1 or in the fragment 2 after the scission, respectively. $T_{\gamma}$ are state dependent energy terms

$$
\begin{aligned}
T_{k(0)}= & \frac{i \hbar}{2}\left(v_{k(0)}^{*} \dot{v}_{k(0)}-\dot{v}_{k(0)}^{*} v_{k(0)}\right)=2\left|v_{k(0)}\right|^{2}\left(\epsilon_{k}-s N_{i_{k}} \lambda\right)-2 G_{k k}\left|v_{k(0)}\right|^{4} \\
& +\frac{\Delta_{k(0)}^{*}}{2}\left(\frac{\left|v_{k(0)}\right|^{4}}{\left(v_{k(0)} u_{k(0)}\right)^{*}}-v_{k(0)} u_{k(0)}\right)+\frac{\Delta_{k(0)}}{2}\left(\frac{\left|v_{k(0)}\right|^{4}}{v_{k(0)} u_{k(0)}}-\left(v_{k(0)} u_{k(0)}\right)^{*}\right)
\end{aligned}
$$


with similar equations for the seniority two configurations. The following notations are used:

$$
\begin{array}{cc}
\Delta_{k(0)}=\sum_{k^{\prime}} \kappa_{k^{\prime}(0)} G_{k k^{\prime}} ; & \Delta_{k(j l)}=\sum_{k^{\prime} \neq j, l} \kappa_{k^{\prime}(j l)} G_{k k^{\prime}} ; \\
\kappa_{k(0)}=u_{k(0)} v_{k(0)} ; & \rho_{k(0)}=\left|v_{k(0)}\right|^{2} ; \\
\kappa_{k(j l)}=u_{k(j l)} v_{k(j l)} ; & \rho_{k(j l)}=\left|v_{k(j l)}\right|^{2} ; \\
P_{0}=\left|c_{0}\right|^{2} ; & P_{j l}=\left|c_{j l}\right|^{2} ; \\
S_{0 j l}=c_{0} c_{j l}^{*} ; & S_{m n j l}=c_{m n} c_{j l}^{*} .
\end{array}
$$

The symbol $\Delta_{\gamma}$ gives the gap parameter. (The label $\gamma$ denotes here generically a specific configuration.) The variables that depend on the time through the generalized coordinates are the single particle densities $\rho_{\gamma}$, the pairing moment components $\kappa_{\gamma}$, the probabilities to have a given seniority configuration $P_{\gamma}$, and the moment components between configurations $S_{\gamma \gamma^{\prime}}$. The relations (4)-(7) are the well known time dependent paring equations for one seniority configuration previously deduced in Refs. $[19,20]$. These formulas are identical to the time dependent Hartree-Fock-Bogoliubov equations [2123]. The symbol $h_{\gamma}$ denotes the Landau-Zener interaction, while $\bar{E}_{\gamma}$ and $T_{\gamma}$ are energy terms. The condition that $\sum_{\gamma} P_{\gamma}=1$ is implicitly ensured through Eqs. (8) and (9) because $\dot{P}_{0}+\sum_{\gamma} \dot{P}_{\gamma}=0$.

\section{The dissipation}

The energy of the nuclear system in the seniority zero state is

$$
\begin{array}{r}
E_{0}=\left\langle\prod_{k}\left(u_{k(0)}(t)+v_{k(0)}(t) a_{k}^{+} a_{k}^{ \pm}\right)|H| \prod_{k}\left(u_{k(0)}(t)+v_{k(0)}(t) a_{k}^{+} a_{k}^{ \pm}\right)\right\rangle \\
=2 \sum_{k} \rho_{k(0)} \epsilon_{k}-\sum_{k} \kappa_{(0)} \sum_{k^{\prime}} \kappa_{k^{\prime}(0)}^{*} G_{k k^{\prime}}-\sum_{k} \rho_{k(0)}^{2} G_{k k} ;
\end{array}
$$

and in the seniority two state is

$$
\begin{array}{r}
E_{j l}=\left\langle a_{j}^{+} a_{l}^{ \pm} \prod_{k \neq j, l}\left(u_{k(j l)}(t)+v_{k(j l)}(t) a_{k}^{+} a_{k}^{ \pm}\right)|H| a_{j}^{+} a_{l}^{ \pm} \prod_{k \neq j, l}\left(u_{k(j l)}(t)+v_{k(j l)}(t) a_{k}^{+} a_{k}^{ \pm}\right)\right\rangle \\
=2 \sum_{k \neq j, l} \rho_{k(j l)} \epsilon_{k}-\sum_{k \neq j, l} \kappa_{k(j l)} \sum_{k^{\prime} \neq j, l} \kappa_{k^{\prime}(j l)}^{*} G_{k k^{\prime}}-\sum_{k \neq j, l} \rho_{k(j l)}^{2} G_{k k}+\epsilon_{j}+\epsilon_{l} .
\end{array}
$$

The corresponding lower energy states $E_{0}^{0}$ and $E_{j l}^{0}$ of the nuclear system in a given configuration are obtained with the previous relations by replacing the densities $\rho_{\gamma}$ and the pairing moment components $\kappa_{\gamma}$ with the adiabatic values obtained in the BCS approximation. Consequently, as defined in Ref. [19], along the fission path the average dissipated energies $E_{\gamma}^{*}$ will be

$$
E_{0}^{*}=E_{0}-E_{0}^{0} ; \quad E_{j l}^{*}=E_{j l}-E_{j l}^{0},
$$

in the seniority zero and the seniority two configurations, respectively. We subtracted from the total potential energy of the nuclear system, its adiabatic value. It was already shown in Ref. [24] that the mean value of the dissipated energy becomes larger when the velocities of the generalized coordinates increase.

\section{Results}

It is possible to solve the equations of motion if the fission trajectory or the potential barrier are provided. In order to determine the fission barriers within the least action principle, two ingredients are 
required: the deformation energy and the inertia. The total energy of the nuclear system is computed in the framework of the macroscopic-microscopic method $[25,26]$. Within this method, the whole system is characterized by some collective coordinates that determine approximately the behavior of many other intrinsic variables. The generalized coordinates vary in time leading to a split of the system. The procedure is analogue to that used in more sophisticated models as the Hartree-Fock, where external constraints simulate the deformation $[27,28]$. The essential idea of our approach is that a macroscopic model, as the liquid drop one, describes quantitatively the smooth trends of the potential energy with respect the particle number and deformation whereas a microscopic approach as the shell model describes local fluctuations. The combined macroscopic-microscopic method should reproduce both smooth trends and local fluctuations. The basic ingredient in such an analysis is the shape parametrization that depends on several macroscopic degrees of freedom. The macroscopic deformation energy is calculated within the liquid drop model. A microscopic potential must be constructed to be consistent with this nuclear shape parametrization. A microscopic correction is then evaluated using the Strutinsky procedure [29].

The basic ingredient of the model is the nuclear shape parametrization. In the following, an axial symmetric nuclear shape surface during the deformation process from one initial nucleus to the separated fragments is obtained by smoothly joining two spheroids of semi-axis $a_{i}$ and $b_{i}(i=1,2)$ with a neck surface generated by the rotation of a circle of radius $R_{3}$ around the axis of symmetry. By imposing the condition of volume conservation we are left by five independent generalized coordinates $\left\{q_{n}\right\}(n=1,5)$ that can be associated to five degrees of freedom: the elongation $R$ given by the distance between the centers of the spheroids; the necking parameter $C=S / R_{3}$ related to the curvature of the neck, the eccentricities $\epsilon_{i}$ associated with the deformations of the nascent fragments and the mass asymmetry parameter $\eta=V_{1} / V_{2}, V_{i}(i=1,2)$ denoting the volumes of the virtual ellipsoids characterized by the semi-axis $a_{i}$ and $b_{i}$. The nuclear shape parametrization is displayed in figure 3 . The entire model can be considered valid as long as the generalized coordinates and their variations in time make sense.

The many-body wave function and the single particle energies are provided by the Woods-Saxon two-center shell model [14]. The Woods-Saxon potential, the Coulomb interaction and the spin orbit term must be diagonalized in an eigenvector basis. The asymmetric two center shell oscillator provides an orthogonal eigenvector basis for only one Hermite space [30-32]. In this Hermite space the behavior of both fragments can be described. When the elongation $R$ is zero, the eigenvector basis becomes that of the anisotropic oscillator. When $R$ tends to infinity, a two oscillator eigenvector system is obtained in the same Hermite space, centered in the two fragments. In the intermediate situation, each eigenfunction has components in the two subspaces that belong to the fragments. So, the two center shell model provides permanently the wave functions associated to the lower energies of the single particle states pertaining to a major quantum number $N_{\max }$. Therefore, molecular states formed by two fragments at scission could be precisely described. Another feature of the two center shell model is related to the localization of the single particle wave function in one of the two potential wells after the scission. As evidenced in Ref. [17], it is possible to predict this localization for a given fragmentation before that the scission is produced. This feature helps us to fix the number of particles in each fragment when Eqs. (4)-(11) are resolved. This model was widely used by the Bucharest group in the calculations addressing the cluster [33, 34] and alpha decay [35-37], the fission [38-40], or the heavy element synthesis [41-43]. For example, the model was able to describe two tangent nuclei in a wide range of mass asymmetries. The half-lives for cluster decay were reproduced. A mechanism for the formation of an $\alpha$-particle on the nuclear surface was supplied. Fission barriers that agree with the evaluated ones were calculated. It was also shown that the two center parametrization is a good approximation for the optimal shapes [44]. Due to these advantages, versions based 


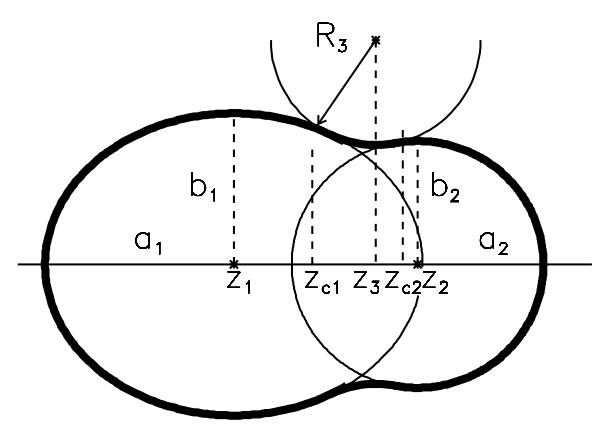

(a) $\mathrm{S}=+1$

(b) $S=-1$

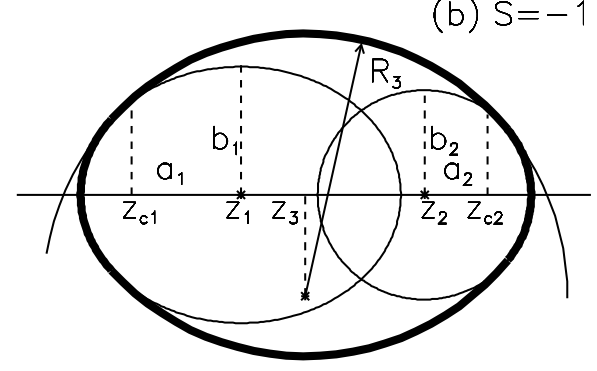

Figure 3. Nuclear shape parametrization. The elongation is defined as $R=z_{2}-z_{1}$. The curvature of the neck parameter is $C=S / R_{3}$, where $S=1$ for necked shapes in the median surface and $S=-1$ otherwise. The eccentricities of the fragments are $\epsilon_{i}=\sqrt{1-\left(b_{i} / a_{i}\right)^{2}}(i=1,2)$. The mass asymmetry parameter can be defined as $\eta=a_{1} b_{1}^{2} /\left(a_{2} b_{2}^{2}\right)$.

on a generalized Nilsson model are widely used in investigating nuclear disintegrations or collisions $[45,46]$.

The fission yields as function of the total excitation energy for the fragmentation ${ }^{90} \mathrm{Kr}+{ }^{144} \mathrm{Ba}$ (even-even), ${ }^{90} \mathrm{Rb}+{ }^{144} \mathrm{Cs}$ (odd-odd) will be investigated in the framework of our model. Experimental data are available for the reaction [7] ${ }^{233} \mathrm{U}\left(\mathrm{n}_{t h}, \mathrm{f}\right)$ where these partitions are investigated. So, two fission channels should be investigated. In the cases of the odd-odd partition, the barrier must be increased with approximately the value of the excitation energy of the unpaired nucleons. In this case, the penetrability is decreased exponentially. The amount of which the barrier is modified can be estimated by accounting the specialization energy. Wheeler defined this specialization energy [47] as the excess of the energy of a nucleon with a given spin over the energy for the same spin nucleon in the state of lowest energy. The least action principle was used to determine the best fission trajectory in our configuration space spanned by our five generalized coordinates. We tested several models for the inertia: the non-adiabatic cranking model [48], the Gaussian overlap approximation $[49,50]$, and the cranking approach [12]. The final results were similar within these different models for the inertia. By minimization, we obtained the most probable fragmentation, that corresponds to a heavy fragment with mass around 138. In our work, the fission path for the 144/90 fragmentation is required. Sophisticated models are used to describe the $Z$ distributions, as for example in Ref. [51]. To overcome these difficulties, some simple assumptions are made. We rely on the results of Refs. $[18,38]$ where the mass distribution of the fragments was relatively well reproduced by considering that the variation of the mass asymmetry is linear from the saddle configuration of the outer barrier up to the exit point. So, it is considered that under the rapid descent from the top of the second barrier different mass partitions are obtained. To obtain these different mass partitions, the simplest way is to vary the mass asymmetry parameter $\eta$ and the averaged deformations of fragments as in Ref. [18]. 


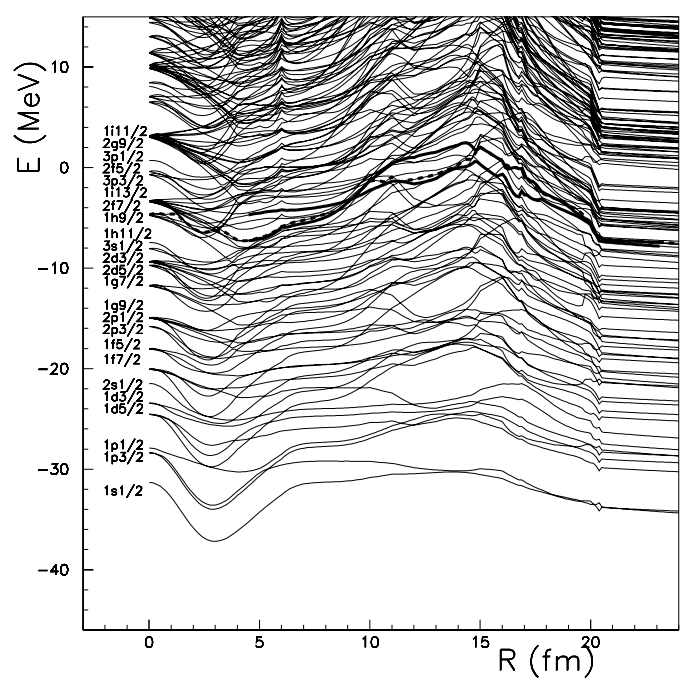

Figure 4. Proton single particle level scheme. The levels with spin projection $\Omega=3 / 2$ that give the lower energy configuration for the unpaired fragments is plotted with a full line. The Fermi energy of the compound nucleus is displayed with a thick dashed line. Four avoided level crossing regions were identified for $R \approx 9.1,14.7,16$ and $20 \mathrm{fm}$.

The penetrabilities for the odd-odd channel is obtained within the same path as that for the even-even channel, but the specialization energy was taken into account.

Once the fission path determined, we need the single particle energies, the variations of the pairing matrix elements $G_{k l}$, the interactions in the avoided crossing regions, and the velocity of passage through these regions in order to solve the equations of motion. In figure 4, the single particle levels obtained within the two center Woods-Saxon model are displayed along the fission trajectory for protons, as an example. The Fermi level of the parent nucleus is plotted with a dashed thick line. The pair of levels with the same spin projection that gives the lower single particle seniority two excitation (or specialization energy) are also displayed with thick full lines. It can be observed that the levels that pertain to the lower excitation are close to the Fermi energy. In the case of protons the spin projection $\Omega$ of these levels is $3 / 2$ while in the case of neutrons it is $\Omega=5 / 2$. These values of $\Omega$ give also the final spin of the partners. Our pairing active space is constructed with 58 single particle levels around the Fermi energy. An important question is the identification of the avoided crossing regions that arise from the strong energy fluctuations of the single particle levels observed along the fission path. For this purpose, we selected all the pairs of adjacent levels with the same spin projection in the active pairing space. From an analyze of the rearrangement of these levels in a manner similar to that of Ref. [52], the avoided crossing regions were identified. In these regions, the excitations are possible between the seniority two and the seniority zero configurations. In other words, these regions are like gates for mixing the configurations, or for changing the seniority. Using the interpolation method described in Ref. [53], the magnitude of the interactions was calculated. Finally, we selected 32 seniority two configurations for protons and 31 configurations for neutrons that are coupled to the seniority zero configuration through avoided levels crossings regions. In the frame of the adiabatic BCS model [12], a mean value of the pairing interaction $G$ can be associated to a given active pairing space, by using a renormalization procedure. So, the values of $G$ for all seniority states were computed for the parent and for the two fragments. A linear interpolation between the values of $G$ from those of the parent to those of the fragments is also realized around the scission point. So, all the most important quantities required to solve the equations of motion are provided: the single particle energies $\epsilon_{k}$, the perturbations $h_{i j}$ and the pairing interactions $G$. The dependence in time is introduced by means of the variations of the collective coordinates. In this respect, several values of the internuclear velocity 

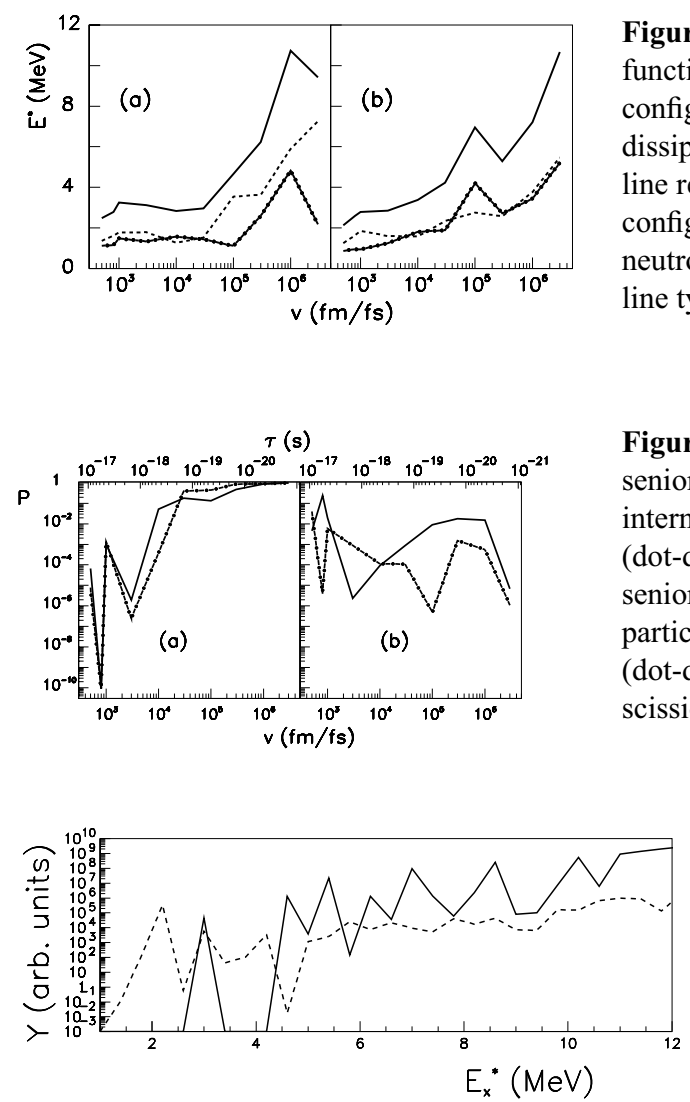

Figure 5. a) Total dissipation energy after the scission $E^{*}$ as function of the internuclear velocity $v$ for the seniority zero configuration. The neutron and proton components of the dissipated energy are plotted with a dot-dashed and a dashed line respectively. (b) Total dissipation for the seniority two configuration with lower single particle excitation. The neutron and proton components are displayed with the same line types as in panel (a).

Figure 6. (a) Probability of realization of the adiabatic seniority zero configuration at scission as function of the internuclear velocity for neutrons (full line) and protons (dot-dashed line). (b) Probability of the realization of the seniority two configuration at scission with the lower single particle excitation for neutrons (full line) and protons (dot-dashed line). The upper axis corresponds to the saddle to scission time $\tau$.

$v=\dot{R}$ are tested in order to solve the equations: $5 \times 10^{2}, 8 \times 10^{2}, 10^{3}, 3 \times 10^{3}, 10^{4}, 3 \times 10^{4}, 10^{5}, 3 \times 10^{5}$, $10^{6}$, and $3 \times 10^{6} \mathrm{fm} / \mathrm{fs}$.

The Equations of motion were solved by taking as initial conditions the BCS ground state values. The final excitation and the probabilities of realizations are plotted in figure 5. The general trend exhibited in both cases is an increase of the final dissipated energy when the internuclear velocity becomes larger. The same behavior is typical for all seniority configurations involved. So the general rule that assesses that the dissipation is proportional to the velocity is retrieved. Another way to introduce the dissipation in quantum systems is to consider forces proportional to the velocity in analogy with the friction forces in classical mechanics [54], leading to a generalized Schrödinger non-linear equation for an open system.

The initial condition for the equations (8)-(11) that describe the configuration mixing is $P_{0}=1$, all the other values being zero. The final probabilities of realization of two configurations for neutrons and protons as function of the internuclear velocities are displayed in Fig. 6. This figure evidences the fact that the probability of realization of adiabatic seniority zero states are close to zero for small velocities, exactly in the energy region in which the dissipation is smaller. So, the main features concerning the relation between the final excitation energy and the probability of realization of a given channel presented in Ref. [15] are retrieved and are valid even if the method for dynamical projection on final atomic and mass numbers is used. The saddle to scission time $\tau$ depends on the intrinsic velocity $v$. This scission time $\tau$ labels the upper axis of Fig. 6 . 
The results concerning the final excitation and the probabilities of realization presented above are coupled through the collective velocity parameter. In order to compare the theoretical findings with the experimental data, this velocity must be eliminated. Therefore, a simple model in accordance to the experimental arrangement is conceived in order to relate the dissipation and the yields. By bombarding the ${ }^{233} \mathrm{U}$ with thermal neutrons, using mass evaluations [55] we found that an excitation of at least $B_{n}=6.85 \mathrm{MeV}$ is accumulated in the compound ${ }^{234} \mathrm{U}$ nucleus. This energy is shared between a potential part $U$ and a kinetic one $T=B_{0} v^{2} / 2$, where $B_{0}$ is the inertia in the ground state configuration. A constant population of all values of the kinetic energy is assumed. The penetrability $P_{b}\left(v, \gamma_{n}, \gamma_{p}\right)$ of each channel depends on the turning points of the barrier, that is, on $v$ through the relation $B_{n}=U+T$. That leads to a larger penetrability when the velocity decreases. The penetrability depends also on the excitation channel $\{\gamma\}$ that can be constructed with the specialization energies of the configurations $\{0\}$ or $\{i j\}$ of the two isospins. The final excitation energy of the two fragments is $E_{x}^{*}\left(v, \gamma_{n}, \gamma_{p}\right)=$ $E^{*}\left(v, \gamma_{n}, \gamma_{p}\right)+E_{s p}\left(\gamma_{n}, \gamma_{p}\right)$, that is, it corresponds to a sum between the dissipated energy $E^{*}$ and the single particle excitations $E_{s p}$ of the two fragments. The dependence of the yield as function of the final excitation $E_{x}^{*}$ in the interval $\left[E_{x 1}^{*}, E_{x 2}^{*}\right]$ exhibits the following proportionality:

$$
\begin{array}{r}
Y_{0}\left(E_{x}^{*}\right) \propto \frac{1}{E_{x 2}^{*}-E_{x 1}^{*}} \int_{0}^{v_{\max }}\left[P_{0(n)}(v) P_{0(p)}(v) P_{b}(v, 0,0)\right. \\
+P_{0(n)}(v) \sum_{\gamma_{p}} P_{\gamma_{p}(p)}(v) P_{b}\left(v, 0, \gamma_{p}\right) \\
+P_{0(p)}(v) \sum_{\gamma_{n}} P_{\gamma_{n}(n)}(v) P_{b}\left(v, \gamma_{n}, 0\right) \\
\left.+\sum_{\gamma_{n}, \gamma_{p}} P_{\gamma_{n}(n)}(v) P_{\gamma_{p}(p)}(v) P_{b}\left(v, \gamma_{n}, \gamma_{p}\right)\right] \\
\left.\times w(v) \theta\left(E_{x 1}^{*}-E_{x}^{*}\left(v, \gamma_{n}, \gamma_{p}\right)\right) \theta\left(E_{x}^{*}\left(v, \gamma_{n}, \gamma_{p}\right)-E_{x 2}^{*}\right)\right) d v,
\end{array}
$$

for the even-even channel. Here $P_{b}$ are the penetrabilities that depend on a specific channel and $P_{0}$ are the probabilities of realization given by the time dependent equations for neutrons (index $(n)$ ) and protons (index $(p))$. For all velocities that give an excitation in the interval $\left[E_{x 1}^{*}, E_{x 2}^{*}\right]$, the previous formula reflects the fact that the yields are proportional to these penetrabilities and probabilities. Of course, at scission it is possible to obtain even-even partitions even if a Cooper pair is broken. In this case, one of the fission products picks this broken pair and will carry a very large excitation energy. Therefore, the sums that run over the channels $\gamma$ in the expression (16) take into consideration the fact that some configurations are formed with a broken pair in only one partner. The probabilities of realization of a given seniority configuration $P_{\gamma_{n}(p)}(v)$ depend on the internuclear velocity. The penetrability of the fission barrier $P_{b}\left(v, \gamma_{n}, \gamma_{p}\right)$ at the velocity $v$ in the channel $\left\{\gamma_{n}, \gamma_{p}\right\}$ depends on the variation of the probabilities of realization along the fission path. The factor $w(v)=B_{0} v$ is a weighting that reflects the dependence of the kinetic energy of the velocity, because $d T=w(v) d v$, and $\theta$ is the step Heaviside distribution used to select only events in the interval $\left[E_{x 1}^{*}, E_{x 2}^{*}\right] . B_{0}$ is considered to be the inertia in the ground state of the parent nucleus. The maximal value of the the velocity is obtained from the boundary $T_{\max }=\frac{1}{2} B_{0} v_{\max }^{2}=B_{n}$. The penetrability $P_{b}\left(v, \gamma_{n}, \gamma_{p}\right)$ is calculated by considering the turning points at the energy $U=B_{n}-\frac{1}{2} B_{0} v^{2}$. The yields for odd-odd partitions are proportional with the expression:

$$
\begin{aligned}
Y_{2}\left(E_{x}^{*}\right) \propto \frac{1}{E_{x 2}^{*}-E_{x 1}^{*}} & \int_{0}^{v_{\max }} \sum_{\gamma_{n}} \sum_{\gamma_{p}} P_{\gamma_{n}(n)}(v,) P_{\gamma_{p}(p)}(v,) P_{b}\left(v, \gamma_{n}, \gamma_{p}\right) w(v) \\
& \times \theta\left(E_{x 1}^{*}-E_{x}^{*}\left(v, \gamma_{n}, \gamma_{p}\right)\right) \theta\left(E_{x}^{*}\left(v, \gamma_{n}, \gamma_{p}\right)-E_{x 2}^{*}\right) d v .
\end{aligned}
$$


In the previous relation, the sums run over all the seniority two configuration taken into consideration for neutrons (index $n$ ) and protons (index $p$ ) that give unpaired nucleons in the two fragments.

The results obtained for the folded distributions $Y_{0}\left(E_{x}^{*}\right)$ and $Y_{2}\left(E_{x}^{*}\right)$ are displayed in figure 7 . These distributions are proportional to the even-even and the odd-odd yields obtained for the fission fragments. The averaging interval is $E_{x 2}^{*}-E_{x 1}^{*}=0.5 \mathrm{MeV}$. The observed experimental trends exhibited in Fig. 4 of Ref. [7] for cold fission yields were reproduced. The trends are same for the three approaches used for the inertia. At low excitation energy the odd-odd yields surpass the even-even ones. The even-even yields become larger for excitation energies larger that 3-4 MeV, in accordance with the experimental findings.

\section{Discussion and conclusions}

In this work, a microscopic model is proposed for the explanation of the odd-even effect in cold fission. This explanation is based on a mixing configuration mechanism that is produced during the fission process. This configuration mixing mechanism is obtained dynamically by solving a the generalized system of time dependent pairing equations, that include a pair-breaking effect. A first rule can be extracted from this model. The even-even fission products cannot be obtained at zero excitation energies because of the existence of dynamical excitations produced in the avoided level crossing regions when the nuclear system deforms slowly.

The magnitudes of the interactions and the location of the the avoided crossing regions are fixed along the fission path and are independent of the velocity of passage through these regions. If this velocity is large, the perturbation will act onto the Cooper pair a small fraction of time. So, the chance to break a pair will be small. If the velocity is low, the pairs will traverse the regions in larger time durations. So, the probability to break a pair increases. On another hand, high velocities lead to large dissipation energies. Another characteristic that was not exploited in this work can be featured from the model. The lower excitation energies of a combination of two odd-odd partners can be obtained only if their spin are the same for neutrons and protons. If the spins of the partners are not the same, the model predicts that at least two pair ruptures are produced for neutrons or protons and additional single particle excitations must be taken into consideration.

The possibility to jump from one level to another in a large scale amplitude motion was predicted by Hill and Wheeler in Ref. [12]. Dissipation in terms of Landau-Zener crossings during fission was first proposed in Ref. [56] where excitations were considered only for time-reversed pairs, neglecting the possible existence of unpaired nucleons. As evidenced in Ref. [57], many studies were performed in order to exploit this mechanism in different type of processes. It was also shown in Ref. [20] that the Landau-Zener mechanism is cached in the time dependent pairing equations (4) and (7). Pairs undergo Landau-Zener transitions on virtual levels with coupling strengths given by the value of the magnitude of the gap parameter. Anyhow, it is the first time that the dynamical pair breaking effect was used to explain the odd-even effect in fission. It must be mentioned that a time-dependent microscopic approach to the scission process was described in Ref. [58]. They observed that for scission time larger than $5 \times 10^{-21} \mathrm{~s}$, the single particle excitations are negligible. This time that characterizes the neck rupture corresponds to a velocity of $10^{6} \mathrm{fm} / \mathrm{fs}$ in our calculations. A typical value for saddle to scission time could be considered as $\tau_{s} \approx 1.3 \times 10^{-21}$. As plotted in Fig. 6, this value of the scission time is consistent with a total excitation energy of the even-even fragments of about $9 \mathrm{MeV}(10 \mathrm{MeV}$ for odd-odd ones). For this value $\tau_{s}$, the probability to have fully paired partners is close to unity.

The density of single particle levels increases in the region of the second barrier. Therefore, from the outer saddle to the scission, many avoided crossing regions are produced and the chance to break a pair is enhanced. Up to the second saddle, the number of avoided crossing regions is small an 
the system evolves merely in the seniority zero configuration. That gives a large penetrability for all channels, the mixing of configurations being produced especially in the outer barrier region.

In this paper, the scission configuration takes in account values for the fragment deformations close to the fundamental ones while the fission path corresponds to cold fission. Therefore, small values of the excitation energy are expected. However, if other deformations of the fragment at scission and a path for more elongated shapes are provided, the same prescriptions can be used for the odd-even effect description. The dissipated energy depends on the modality in which the microscopic levels are rearranged along the specific fission trajectory. If the deformations at scission differ from the ground state ones, the specialization energy and the deformation energy of each fragment increase in magnitude giving finally larger excitation energies.

In conclusion, by solving the dynamical microscopic equations of motion for an fissioning eveneven system it is found that the probability to obtain an odd-odd partition overcomes the probability of an even-even one at excitation energies smaller than $4 \mathrm{MeV}$, for the same division in mass numbers. The theoretical results are in accordance with the experimental behavior of the odd-even distributions at high kinetic energies. It is the first time that this behavior was explained within a quantum mechanical approach.

Acknowledgements Work supported by CNCS-UEFISCDI, project number PN-II-ID-PCE-20113-0068.

\section{References}

[1] W.N. Reisdorf, J. P. Unik, and L. E. Glendenin, Nucl. Phys. A 205, 348 (1973)

[2] H. Wohlfarth, W. Lang, H.-G. Clerc, H. Schrader, K.-H. Schmidt, and H. Dann, Phys. Lett. B 63, 275 (1976)

[3] C. Signarbieux, M. Montoya, M. Ribrag, C. Mazur, C. Guet, P. Perrin, and M. Maurel, J. Phys. Lett. 42, 437 (1981)

[4] M. Caamano, F. Rejmund, and K.-H. Schmidt, J. Phys. G 38, 035101 (2011)

[5] B. Jurado and K.-H. Schmidt, EPJ Web of Conferences 62, 07003 (2013)

[6] H.-G. Clerc, in Heavy Elements and Related New Phenomena, edited by W. Greiner and R. K. Gupta (World Scientific, Singapore, 1999), p. 471

[7] E. Schwab, H.-G. Clerc, M. Mutterer, J. P. Theobald, and H. Faust, Nucl. Phys. A 577, 674 (1994)

[8] F.-J. Hambsch, H.-H. Knitter, and C. Budtz-Jorgensen, Nucl. Phys. A 554, 209 (1993)

[9] H.-H. Knitter, F.-J. Hambsch, and C. Budtz-Jorgensen, Nucl. Phys. A 536, 221 (1992)

[10] V.Avrigeanu, A. Florescu, A. Sandulescu, and W. Greiner, Phys. Rev. C 52, R1755 (1995)

[11] B. Jurado and K.-H. Schmidt, J. Phys. G. 42, 055101 (2015)

[12] D. L. Hill and J. A. Wheeler, Phys. Rev. 89, 1102 (1953)

[13] F. Gonnenwein, Phys. Procedia 47, 107 (2013)

[14] M. Mirea, Phys. Rev. C 78, 044618 (2008)

[15] M. Mirea, Phys. Lett. B 680, 316 (2009)

[16] M. Mirea, Phys. Rev. C 89, 034623 (2014)

[17] M. Mirea, Phys. Rev. C 83, 054608 (2011)

[18] M. Mirea, Phys. Lett. B 717, 252 (2012)

[19] S.E. Koonin, J.R. Nix, Phys. Rev. C 13, 209 (1976)

[20] J. Blocki, H. Flocard, Nucl. Phys. A 273, 45 (1976)

[21] B. Avez, C. Simenel and Ph. Chomaz, Phys. Rev. C 78, 044318 (2008) 
[22] S. Ebata, T. Nakatsukasa, T. Inakura, K. Yoshida, Y. Hashimoto and K. Yabana, Phys. Rev. C 82, $034306(2010)$

[23] J.W. Negele, Rev. Mod. Phys. 54, 913 (1982).

[24] M. Mirea, L. Tassan-Got, C. Stephan, C.O. Bacri, P. Stoica, R.C. Bobulescu, Nucl. Phys. A 735, 21 (2004).

[25] J.R. Nix, Ann. Rev. Nucl. Sci. 22, 65 (1972).

[26] W.J. Swiatecki, S. Bjornholm, Phys. Rep. 4, 325 (1972).

[27] C. Simenel, A.S. Umar, Phys. Rev. C 89, 031601(R) (2014).

[28] G. Scamps, C. Simenel, D. Lacroix, Phys. Rev. C 92, 011602(R) (2015).

[29] M. Brack, J. Damgaard, A.S. Jensen, H.C. Pauli, V.M. Strutinsky, C.Y. Wong, Rev. Mod. Phys. 44, 320 (1972).

[30] J. Maruhn, W. Greiner, Z. Phys. 251, 431 (1972).

[31] M. Mirea, Phys. Rev. C 54, 302 (1996).

[32] M. Mirea, Nucl. Phys. A 780, 13 (2006).

[33] M. Mirea, A. Sandulescu, D.S. Delion, Nucl. Phys. A 870, 23 (2011).

[34] M. Mirea, A. Sandulescu, D.S. Delion, Eur. Phys. J. A 48, 85 (2012).

[35] A. Sandulescu, M. Mirea, D.S. Delion, EPL 101, 62001 (2013).

[36] M. Mirea, Eur. Phys. J. A 51, 36 (2015).

[37] M. Mirea, Rom. J. Phys. 60, 156 (2015).

[38] M. Mirea, D.S. Delion, A. Sandulescu, Phys. Rev. C 81, 044317 (2010).

[39] M. Mirea, L. Tassan-Got, Centr. Eur. J. Phys. 9, 116 (2011).

[40] R. Budaca, A. Sandulescu, M. Mirea, Mod. Phys. Lett. A 30, 1550129 (2015).

[41] M. Mirea, D.S. Delion, A. Sandulescu, EPL 85, 121001 (2009).

[42] A. Sandulescu, M. Mirea, Eur. Phys. J. A 50, 110 (2014).

[43] D. Aranghel and A. Sandulescu, Rom. J. Phys. 60, 147 (2015).

[44] F.A. Ivanyuk, S. Chiba, A. Arimoto, Phys. Rev. C 90, 054607 (2014).

[45] A. Diaz-Torres, W. Scheid, Nucl. Phys. A 757, 373 (2005).

[46] Y. Arimoto, S. Chiba, Phys. Rev. C 88, 044614 (2013).

[47] J.A. Wheeler, Niels Bohr and the Development of Physics, edited by W. Pauli, L. Rosenfeld, and W. Weisskopf (Pergamon Press, London, 1955) p. 163.

[48] M. Mirea, R.C. Bobulescu, J. Phys. G 37, 055106 (2010).

[49] A. Gozdz, K. Pomorski, M. Brack, E. Werner, Nucl. Phys. A 442, 26 (1985).

[50] A. Gozdz, K. Pomorski, M. Brack, E. Werner, Nucl. Phys. A 442, 50 (1985).

[51] J. Randrup, P. Moller, Phys. Rev. Lett. 106, 132503 (2011).

[52] M. Mirea, L. Tassan-Got, C. Stephan, C.O. Bacri and R.C. Bobulescu, Phys. Rev. C 76, 064608 (2007).

[53] W. Greiner, J.Y. Park, and W. Scheid, Nuclear Molecules (World Scientific, Singapore, 1995).

[54] A. Sandulescu, H. Scutaru, Ann. Phys. 173, 277 (1987).

[55] G. Audi, A.H. Wapstra, C. Thibault, Nucl. Phys. A 729, 337 (2003).

[56] G. Schutte, L. Wilets, Z. Phys. A 286, 313 (1978).

[57] R.W. Hasse, Rep. Prog. Phys. 41, 1027 (1978).

[58] M. Rizea, N. Carjan, Proc. Rom. Acad. Series A 16,176 (2015). 